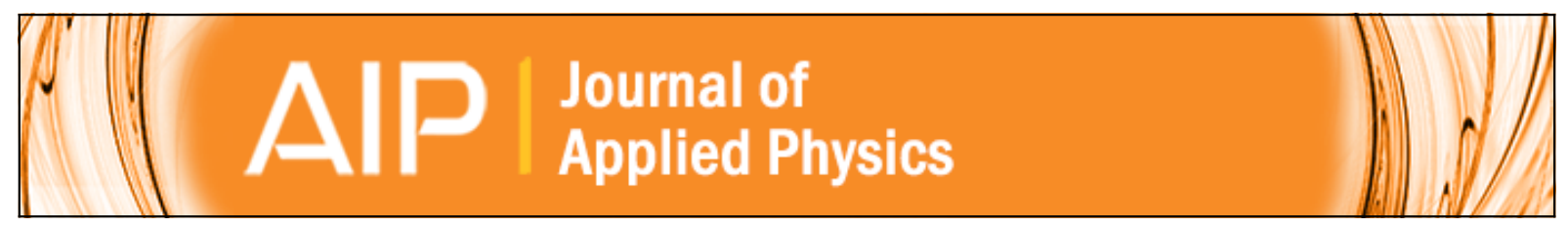

\title{
Electron transport across a Gaussian superlattice
}

I. Gómez, F. Domnguez-Adame, E. Diez, and V. Bellani

Citation: Journal of Applied Physics 85, 3916 (1999); doi: 10.1063/1.369764

View online: http://dx.doi.org/10.1063/1.369764

View Table of Contents: http://scitation.aip.org/content/aip/journal/jap/85/7?ver=pdfcov

Published by the AIP Publishing

\section{Articles you may be interested in}

Nonlinear superlattice transport limited by Joule heating

J. Appl. Phys. 92, 6043 (2002); 10.1063/1.1510567

Miniband transport in vertical superlattice field-effect transistors

Appl. Phys. Lett. 79, 1564 (2001); 10.1063/1.1390320

Far-infrared electroluminescence from parabolic quantum well superlattices excited by resonant tunneling injection

J. Appl. Phys. 88, 172 (2000); 10.1063/1.373638

Controllable bistabilities and bifurcations in a photoexcited GaAs/AIAs superlattice

Appl. Phys. Lett. 74, 3845 (1999); 10.1063/1.124199

Millimeter wave oscillator based on a quasiplanar superlattice electronic device

Appl. Phys. Lett. 71, 401 (1997); 10.1063/1.119550

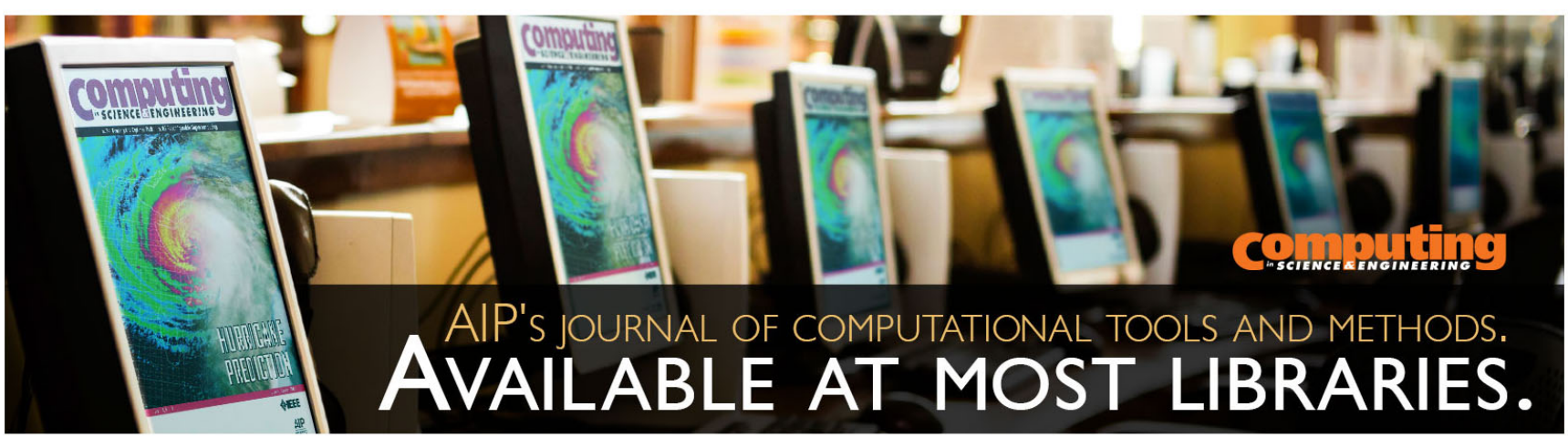




\section{COMMUNICATIONS}

\section{Electron transport across a Gaussian superlattice}

I. Gómez and F. Domínguez-Adame ${ }^{\mathrm{a})}$

GISC, Departamento de Física de Materiales, Universidad Complutense, E-20840 Madrid, Spain

E. Diez

GISC, Departamento de Matemáticas, Universidad Carlos III de Madrid, E-28911 Leganés, Madrid, Spain

V. Bellani

INFM and Dipartimento di Fisica "A. Volta," Universitá di Pavia, I-27100 Pavia, Italy

(Received 28 September 1998; accepted for publication 21 December 1998)

We study the electron transmission probability in semiconductor superlattices where the height of the barriers is modulated by a Gaussian profile. Such structures act as efficient energy band-pass filters and, contrary to previous designs, it is expected to present a lower number of unintentional defects and, consequently, better performance. The $j-V$ characteristic presents negative differential resistance with peak-to-valley ratios much greater than in conventional semiconductor superlattices.

(C) 1999 American Institute of Physics. [S0021-8979(99)06506-8]

Quantum mechanics has a substantial impact in modern solid-state electronics. The occurrence of energy minibands separated by minigaps in a semiconductor superlattice (SL) is a good example. The spatial overlap between electronic states of neighboring wells shifts the degenerate energy levels and lead to the appearance of minibands. Recent developments in epitaxial growth technology have made possible to tailor the band structure of SLs to nearly arbitrary precision. The resulting band structure strongly depends on the potential profile and a variety of them have been proposed (square, sawtooth, parabolic). Electron transport in SLs was first considered by Esaki and Tsu, ${ }^{1}$ who reported that a region of negative differential resistance (NDR) should occur. Furthermore, if the length of the SL is shorter than the carrier mean free path, coherent transmission through an ideal SL is possible. ${ }^{2}$ In this context, Tung and $\mathrm{Lee}^{3}$ has recently proposed a novel quantum mechanical energy band-pass filter using SLs based on asymmetric quantum wells (QWs). Such structures present a Gaussian potential profile and allow the incident electrons to be nearly unscattered when the impinging electron energy lies in the band pass. This is quite different from the transmission characteristic in uniform SLs with Kronig-Penney profile, where the transmission probability is not uniformly equal to unity within each miniband.

The band-pass filter proposed in Ref. 3 is a QW-based SL whose conduction-band edge is modulated by a Gaussian function. The top of the barrier centered at $x_{b}$ (here $x$ denotes the spatial coordinate along the growth direction) is set according to the function $V_{0} \exp \left(-x_{b}^{2} / \sigma^{2}\right)$, where $V_{0}$ is constant and $\sigma / \sqrt{2}$ is the standard deviation of the Gaussian function. Similarly, the bottom of the well centered at $x_{w}$ is chosen as $-V_{0} \exp \left(-x_{w}^{2} / \sigma^{2}\right)$. This potential profile is obtained by

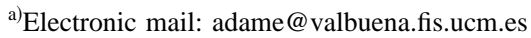

changing the mole fraction in $\mathrm{Al}_{x} \mathrm{Ga}_{1-x} \mathrm{As}$ material system. Note that both the barriers and the wells are modulated, that is, the mole fraction varies in every layer of the SL. In particular, the barrier height of the central barrier is $2 V_{0}$ above from the bottom of the adjacent well. A typical value proposed in Ref. 3 is $V_{0}=0.45 \mathrm{eV}$, which would require prohibitively high values of the mole fraction from the experimental point of view. Such high values lead to a number of growth defects which would make the device unreliable (recall that disorder leads to localization of electrons and hence to a reduction of the transmission probability). ${ }^{4}$

The aim of this article is twofold. First, we present a new model of band-pass filter where only the barriers are modulated, whereas the wells are made of the same semiconductor (say GaAs). We expect this setup will present better crystallographic quality than that proposed by Tsung and $\mathrm{Lee}^{3}$ and, consequently, higher efficiency in actual devices. Second, we study the $j-V$ characteristic and find NDR with a peak-tovalley ratio several orders of magnitude higher than in uniform SLs.

We consider a GaAs- $\mathrm{Al}_{x} \mathrm{Ga}_{1-x}$ As SL. For our present purposes, it is enough to focus on electron states close to the conduction-band edge and use the effective mass approximation. For simplicity we consider a uniform effective mass at the $\Gamma$ valley throughout the structure $\left(m^{*}=0.067 m, m\right.$ being the free electron mass). Moreover, we take the momentum parallel to the layers equal to zero. We focus on electron states close to the band gap and use the one-band effectivemass framework to calculate the envelope functions

$$
\left[-\frac{\hbar^{2}}{2 m^{*}} \frac{d^{2}}{d x^{2}}+V_{\mathrm{SL}}(x)\right] F(x)=E F(x),
$$

where $V_{\mathrm{SL}}(x)$ is the conduction-band profile. As it was mentioned above, the height of the barrier centered at $x_{b}$ is 

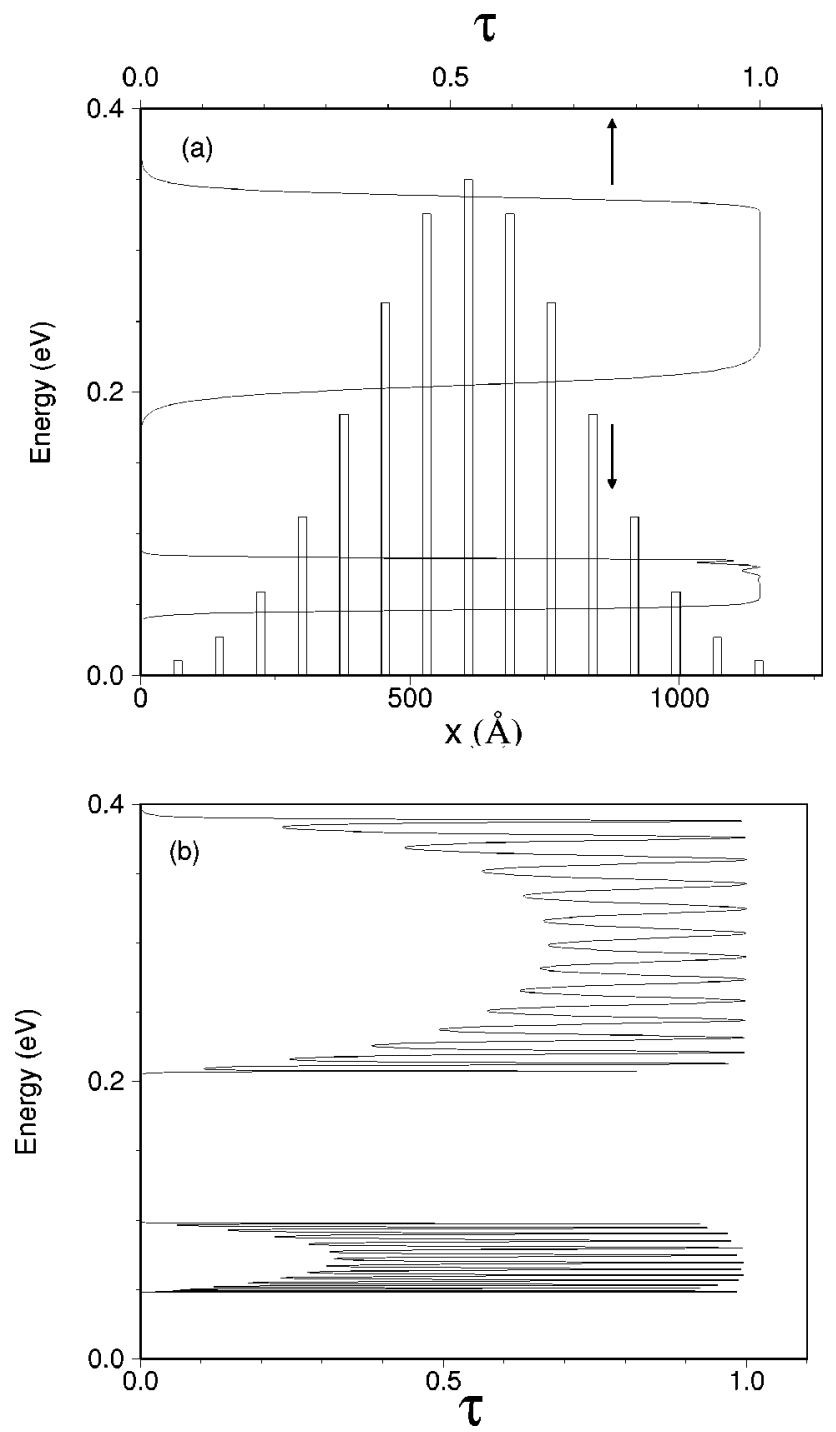

FIG. 1. (a) Plots of the Gaussian SL potential profile and transmission probability. Notice the occurrence of two bands below the highest barrier. (b) Plot of the transmission probability for a uniform SL with the same number of barriers.

$V_{0} \exp \left(-x_{b}^{2} / \sigma^{2}\right)$ but the bottom of every well lies at the same energy, which we will take as the origin of energy. In our calculations we have taken $V_{0}=0.35 \mathrm{eV}$. The width of the barriers is $1.5 \mathrm{~nm}$, the width of the wells is $6.2 \mathrm{~nm}$, and the parameter $\sigma$ is $28.875 \mathrm{~nm}$. Figure 1(a) shows the conduction band edge of the investigated SL with 15 barriers.

The corresponding envelope-function values at both sides of the $n$th barrier are related via a $2 \times 2$ transfer matrix $M(n)$ whose elements can be cast in the form $M_{11}(n)$ $=M_{22}^{*}(n) \equiv \alpha_{n}$ and $M_{12}(n)=M_{21}^{*}(n) \equiv \beta_{n}$ (see, e.g., Refs. 5 and 6). Letting $N$ be the total number of barriers, the transfer matrix of the SL is obtained as the product $T(N)$ $=M(N) M(N-1) \ldots M(1)$. The element $T_{11}(N) \equiv A_{N}$ can be easily calculated recursively from the relationship ${ }^{6}$

$$
A_{n}=\left(\alpha_{n}+\alpha_{n-1}^{*} \frac{\beta_{n}}{\beta_{n-1}}\right) A_{n-1}-\left(\frac{\beta_{n}}{\beta_{n-1}}\right) A_{n-2},
$$

supplemented by the initial conditions $A_{0}=1, A_{1}=\alpha_{1}$. The knowledge of $A_{N}$ enables us to obtain the transmission coef- ficient $\tau$ at a given energy $E, \tau=\left|A_{N}\right|^{-2}$. The calculated transmission probability is plotted as a function of the incident energy $E$ in Fig. 1(a). Notice the occurrence of two bands below the highest barrier $(0.35 \mathrm{eV})$. The value of the transmission probability for energies within these two bands is almost equal to unity. This is a remarkable characteristic of this devices because the number of barriers is rather small. The uniform (i.e., Kronig-Penney) SL with the same parameters also presents two bands with energy below $V_{0}$ but, contrary to the Gaussian SL, the transmission probability is not constant but oscillates as a function of energy [see Fig. 1(b)].

To seek a physical magnitude amenable to experimental verification, we have numerically evaluated the $j-V$ characteristic of both kinds of SLs. The current density at a given temperature $T$ for a SL subject to a uniform electric field can be calculated within the stationary-state $\operatorname{model}^{7}$ (see also Ref. 8 for further details)

$$
j(V)=\frac{m^{*} e k_{B} T}{2 \pi^{2} \hbar^{3}} \int_{0}^{\infty} N(E, V) \tau(E, V) d E,
$$

where $V$ is the applied bias and $k_{B}$ the Boltzmann constant. $N(E, V)$ accounts for the occupation of states in both sides of the device, according to the Fermi distribution function, and it is given by

$$
N(E, V)=\ln \left(\frac{1+\exp \left[\left(E_{F}-E\right) / k_{B} T\right]}{1+\exp \left[\left(E_{F}-E-e V\right) / k_{B} T\right]}\right),
$$

$E_{F}$ being the Fermi level, which will be assumed to be located at the conduction-band edge of GaAs in what follows. The transmission probability $\tau(E, V)$ at a given bias $V$ is obtained by discretizing the effective-mass Eq. (1) for the SL potential $V_{\mathrm{SL}}(x)$ plus the linear potential $-e V x / L$ due to the applied electric field, ${ }^{9}$ where $L$ is the SL length. The electric field is assumed to be applied only on the SL whereas it vanishes at the contacts. We divide the interval $[0, L]$ in a grid of points $\left\{x_{k}=k s\right\}$, where $s$ is the integration step. The discretized form of the effective-mass Eq. (1) may be cast in the matrix form

$$
\left(\begin{array}{c}
F\left(x_{k+1}\right) \\
F\left(x_{k}\right)
\end{array}\right)=\left(\begin{array}{cc}
\alpha_{k} & -1 \\
1 & 0
\end{array}\right)\left(\begin{array}{c}
F\left(x_{k}\right) \\
F\left(x_{k-1}\right)
\end{array}\right) \equiv P_{k}\left(\begin{array}{c}
F\left(x_{k}\right) \\
F\left(x_{k-1}\right)
\end{array}\right),
$$

where $\alpha_{k} \equiv 2+\left(2 m^{*} s^{2} / \hbar^{2}\right)\left[V_{\mathrm{SL}}\left(x_{k}\right)-e V x_{k} / L-E\right]$. This form is suitable for a transfer-matrix approach to solve the scattering problem. In fact, iterating this equation one obtains

$$
\left(\begin{array}{c}
F\left(x_{N_{s}+1}\right) \\
F\left(x_{N_{s}}\right)
\end{array}\right)=P_{N_{s}} \cdots P_{0}\left(\begin{array}{c}
F\left(x_{0}\right) \\
F\left(x_{-1}\right)
\end{array}\right) \equiv T\left(N_{s}\right)\left(\begin{array}{c}
F\left(x_{0}\right) \\
F\left(x_{-1}\right)
\end{array}\right) .
$$

$T\left(N_{s}\right)$ is the global transfer matrix and $N_{s}$ is the number of grid points in the SL. $T\left(N_{s}\right)$ is real and relates the wave function at both edges of the structure. The solution of the wave equation is the field-free region is given by

$$
F\left(x_{k}\right)=\left\{\begin{array}{l}
e^{i q_{l} k}+r e^{-i q_{l} k} \quad k \leqslant 0, \\
t e^{i q_{r} k} \quad k \geqslant N_{s},
\end{array}\right.
$$




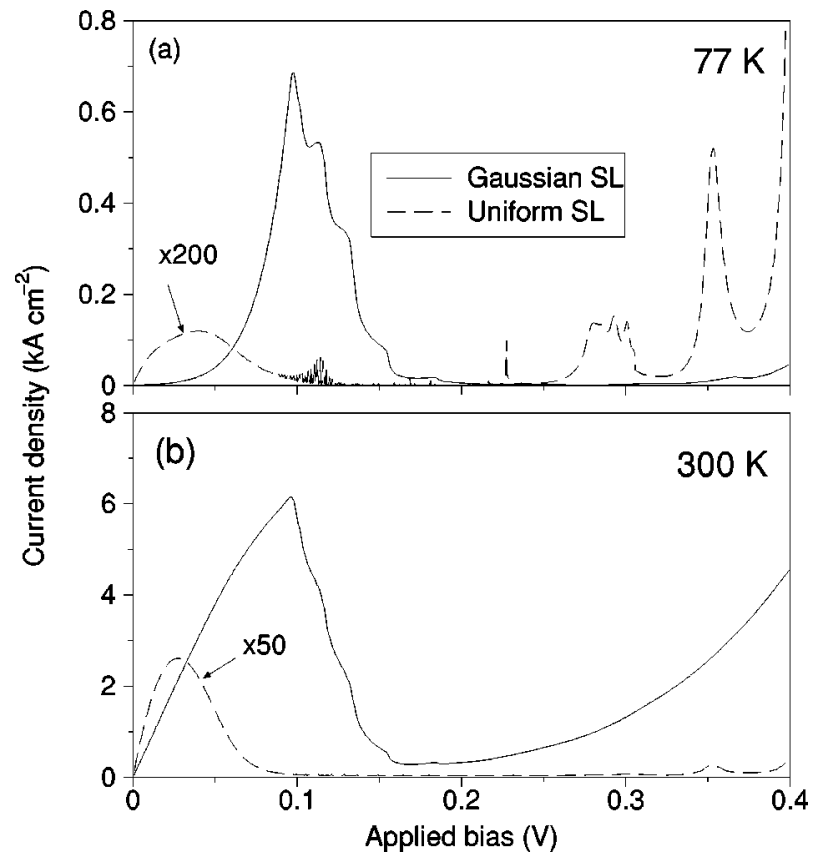

FIG. 2. $j-V$ characteristic of Gaussian (solid lines) and uniform (dashed lines) SLs at (a) 77 and (b) $300 \mathrm{~K}$.

where $q_{l} \equiv \sqrt{2 m^{*} E s^{2} / \hbar^{2}}$ and $q_{r} \equiv \sqrt{2 m^{*}(E+e V) s^{2} / \hbar^{2}}$ for small $s$. Using Eqs. (6) and (7), we find that the transmission coefficient is given by

$$
\tau(E, V)=\frac{4 \sin q_{l} \sin q_{r}}{D(E, V)},
$$

with

$$
\begin{aligned}
D(E, V) \equiv & T_{11}^{2}+T_{12}^{2}+T_{21}^{2}+T_{22}^{2} \\
& +2\left(T_{11} T_{12}+T_{21} T_{22}\right) \cos q_{l} \\
& -2\left(T_{11} T_{21}+T_{12} T_{22}\right) \cos q_{r}-2\left(T_{11} T_{22}\right. \\
& \left.+T_{12} T_{21}\right) \cos q_{l} \cos q_{r}+2 \sin q_{l} \sin q_{r},
\end{aligned}
$$

where the dependence of $T_{i j}$ on $N_{s}$ has been omitted for brevity. Taking into account that $T(k)=P_{k} T(k-1)$ and $T(0)=P_{0}$ we find the following recurrence relation:

$$
\begin{aligned}
& T_{11}(k)=\alpha_{k} T_{11}(k-1)-T_{11}(k-2), \\
& T_{12}(k)=\alpha_{k} T_{12}(k-1)-T_{12}(k-2), \\
& T_{21}(k)=T_{11}(k-1), \\
& T_{22}(k)=T_{12}(k-1), k=1,2 \cdots N_{s} .
\end{aligned}
$$

In order to determine the transmission coefficient, these equations must be supplemented with the initial conditions $T_{i j}(-1)=\delta_{i j}, T_{11}(0)=\alpha_{0}, T_{12}(0)=-1, T_{21}(0)=1$ and $T_{22}(0)=0$. After the transmission coefficient has been determined numerically, we calculate the $j-V$ characteristic from Eq. (3). We show in Fig. 2 the $j-V$ characteristic for both Gaussian and uniform SLs at two different temperatures (77 and $300 \mathrm{~K}$ ). We observe the occurrence of NDR in both SLs, but the corresponding peak-to-valley ratios are quite different. In particular, the Gaussian SL displays peak-to-valley ratios 40 and 20 at 77 and $300 \mathrm{~K}$, respectively. It is important to mention that these results are based on the assumption of purely ballistic current regime across the SL. In a recent article, Rauch et al. ${ }^{10}$ have found that the coherence length in uniform GaAs- $\mathrm{Al}_{x} \mathrm{Ga}_{1-x} \mathrm{As}$ is about $150 \mathrm{~nm}$, namely larger than the SL length we have studied $(110 \mathrm{~nm})$. Therefore, we can confidently assume coherent transport across the SL.

To conclude, we have presented a new design of a bandpass filters using SLs. We propose to modulate the barrier heights with a Gaussian function, whereas the bottom of the QWs remain unchanged. The obtained transmission probability is almost equal to unity within the allowed minibands. Contrary to previous devices based on $\mathrm{SL},{ }^{3}$ in the present $\mathrm{SL}$ the mole fraction varies in the barriers but not in the wells, allowing for a better crystallographic quality of the structure and, consequently, leading to higher performance. We have presented calculations of the $j-V$ characteristic and found NDR in both Gaussian and uniform SLs. However, the current density attained in Gaussian SLs is several orders of magnitude larger than in uniform SLs for the same structural parameters. This result allows us to experimentally verify our predictions. Work along this direction is currently in progress.

Work at Madrid has been supported by CAM (Spain) under Project No. 07N/0034/1998.

\footnotetext{
${ }^{1}$ L. Esaki and R. Tsu, IBM J. Res. Dev. 14, 61 (1970).

${ }^{2}$ R. Tsu and L. Esaki, Appl. Phys. Lett. 22, 562 (1973).

${ }^{3}$ H.-H. Tung and C.-P. Lee, IEEE J. Quantum Electron. 32, 507 (1996).

${ }^{4}$ K. A. Mäder and A. Zunger, Europhys. Lett. 31, 107 (1995).

${ }^{5}$ J. H. Luscombe, Nanotechnology 4, 1 (1993).

${ }^{6}$ F. Domínguez-Adame, A. Sánchez, and E. Diez, Phys. Rev. 50, 17736 (1994).

${ }^{7}$ C. B. Duke, Tunneling in Solids (Academic, New York, 1969).

${ }^{8}$ T. B. Boykin, Phys. Rev. B 51, 4289 (1995).

${ }^{9}$ F. Domínguez-Adame, B. Méndez, and E. Maciá, Semicond. Sci. Technol. 9, 263 (1994).

${ }^{10}$ C. Rauch, G. Strasser, K. Unterrainer, W. Boxleitner, and G. Gornik, Phys. Rev. Lett. 81, 3495 (1998).
} 